\title{
Tifus de los matorrales, una enfermedad emergente en Chile
}

\author{
Katia Abarca1,2, Thomas Weitzeß', Constanza Martínez-Valdebenito ${ }^{1,2}$ y Gerardo Acosta-Jamett4
}

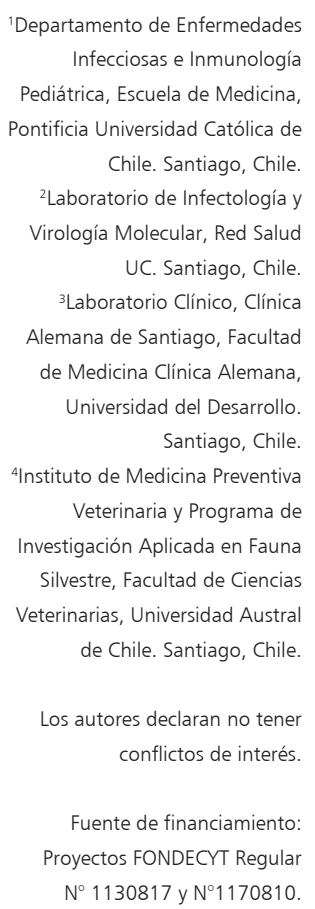

Gerardo Acosta J. gerardo.acosta@uach.cl

\section{Introducción}

E 1 reconocimiento de una nueva enfermedad infecciosa en un país constituye un desafío diagnóstico por la falta de reconocimiento por los médicos clínicos y la ausencia de pruebas diagnósticas. En el año 2006 se diagnosticó por primera vez en Chile un caso de tifus de los matorrales en un biólogo que regresaba de trabajo en terreno cerca de Ancud, Chiloé1. Su presentación clásica de una rickettsiosis (fiebre, exantema, aumento de transaminasas hepáticas) con mancha negra y la detección de material genético de Orientia sp. constituyó una novedad, puesto que en Chile no se presentaba una rickettsiosis desde inicios del siglo $\mathrm{XX}$, cuando se presentaron brotes de tifus epidémico $(R \text {. prowazekii })^{2}$, transmitido por el piojo corporal, un ectoparásito común en esos tiempos en Chile.

El tifus de los matorrales causado por una rickettsia denominada Orientia tsutsugamushi, es una enfermedad endémica en una zona del Asia Pacífico llamada triángulo tsutsugamushi, y está asociada a la ruralidad y a la pobreza y afecta a un millón de personas cada $a \tilde{n} 0^{3-5}$. Por sus características epidemiológicas y clínicas es considerada como la enfermedad prevalente, sub reconocida, grave y fácilmente tratable del mundo ${ }^{6,7}$. Es transmitida por la mordedura indolora y, por tanto, inadvertida de larvas de ácaros ambientales de la familia Trombiculidae, conocidos como ácaros de la cosecha o ácaros rojos (en inglés: chigger mites $)^{8}$. Clásicamente se presenta como un cuadro febril asociado a exantema y una costra negra o escara en la zona de inoculación ${ }^{3,7,9}$. La escara se constituye unos días posteriores a la inoculación, pasando por un estado inicial de pápula eritematosa que se va necrosando en el centro, rodeada a veces de un halo eritematoso. La lesión es pequeña, del tamaño de una quemadura de cigarro y no es dolorosa ${ }^{10}$. La fiebre aparece uno a dos días después, en forma abrupta, suele ser alta, acompañada de compromiso del estado general, cefalea intensa, dolor retro-ocular, mialgias y sudoración nocturna. Puede haber congestión conjuntival, artralgias, tos, dolor abdominal, disminución de la agudeza auditiva y un compromiso del sensorio que le da el nombre de tifus a la enfermedad (del griego typhos, "estupor que como el humo oscurece y nubla la mente") $)^{3,7,9}$. En los exámenes de laboratorio suele haber aumento de los reactantes de fase aguda y un aumento leve a moderado de las transaminasas hepáticas; puede presentarse también leucopenia, trombocitopenia y aumento de $\mathrm{LDH}^{11}$.

Como en otras infecciones rickettsiales, la rápida recuperación de la fiebre y los síntomas constitucionales tras el inicio de la terapia con doxiciclina puede ser considerada una prueba terapéutica ${ }^{3,12,13}$. Sin tratamiento, la defervescencia ocurre en una a dos semanas, pero se han descrito complicaciones que pueden llevar a falla multiorgánica y muerte ${ }^{5,14}$. Las complicaciones más frecuentes son neumonía atípica, insuficiencia renal y meningoencefalitis ${ }^{14}$. La mortalidad sin tratamiento es de $6 \%$ (mediana, con un rango de 1 a 70\%), de 1,6\% en los casos tratados (rango 0-33\%) y hasta $13 \%$ en áreas con posible resistencia a las terapias habituales ${ }^{15,16}$.

A partir del año 2015 y hasta abril de 2018, como resultado de dos proyectos de investigación (uno aún en 
curso) nuestro grupo ha identificado más de 30 pacientes que cumplen criterios clínicos de tifus de los matorrales; algunos de ellos ya han sido confirmados y otros se encuentran en estudio, siendo progresivo el número de casos identificados cada año. Todos los casos han ocurrido en meses de verano.

Como en los primeros casos publicados ${ }^{17}$, la gran mayoría de los casos identificados o en estudio, han sido identificados en Chiloé, principalmente en la zona norte de la isla. La determinación del rango de distribución espacial es parte de los objetivos del proyecto actual; datos preliminares sugieren que la infección también se presenta en Chile continental, probablemente incluyendo las regiones de Los Lagos y Aysén. Notablemente, en 2018 se detectaron varios casos en la Región Metropolitana, en turistas que visitaron las zonas endémicas del sur del país. Por ello es relevante que todos los médicos en Chile conozcan la presentación clínica de esta nueva infección y los principios de su manejo clínico.

Los casos detectados tienen un predominio de varones, con contacto laboral o recreacional (ej. senderismo y acampada) con vegetación o leña en zonas rurales. Los casos han sido en su gran mayoría muy característicos, presentándose mayoritariamente con la tríada fiebre, exantema y escara (Figura 1, A-G). Entre los otros síntomas destaca, por su alta frecuencia, la cefalea y la sudoración nocturna. En el laboratorio ha predominado un aumento de la proteína C reactiva (PCR) y de transaminasas hepáticas. Afortunadamente, hasta ahora no hemos detectado casos graves ni mortalidad. Sin embargo, una buena proporción de los pacientes ha consultado repetidamente, sin que el médico sospeche de esta entidad. Las hipótesis diagnósticas inicialmente planteadas han sido loxoscelismo, celulitis, reacciones alérgicas, mononucleosis infecciosa

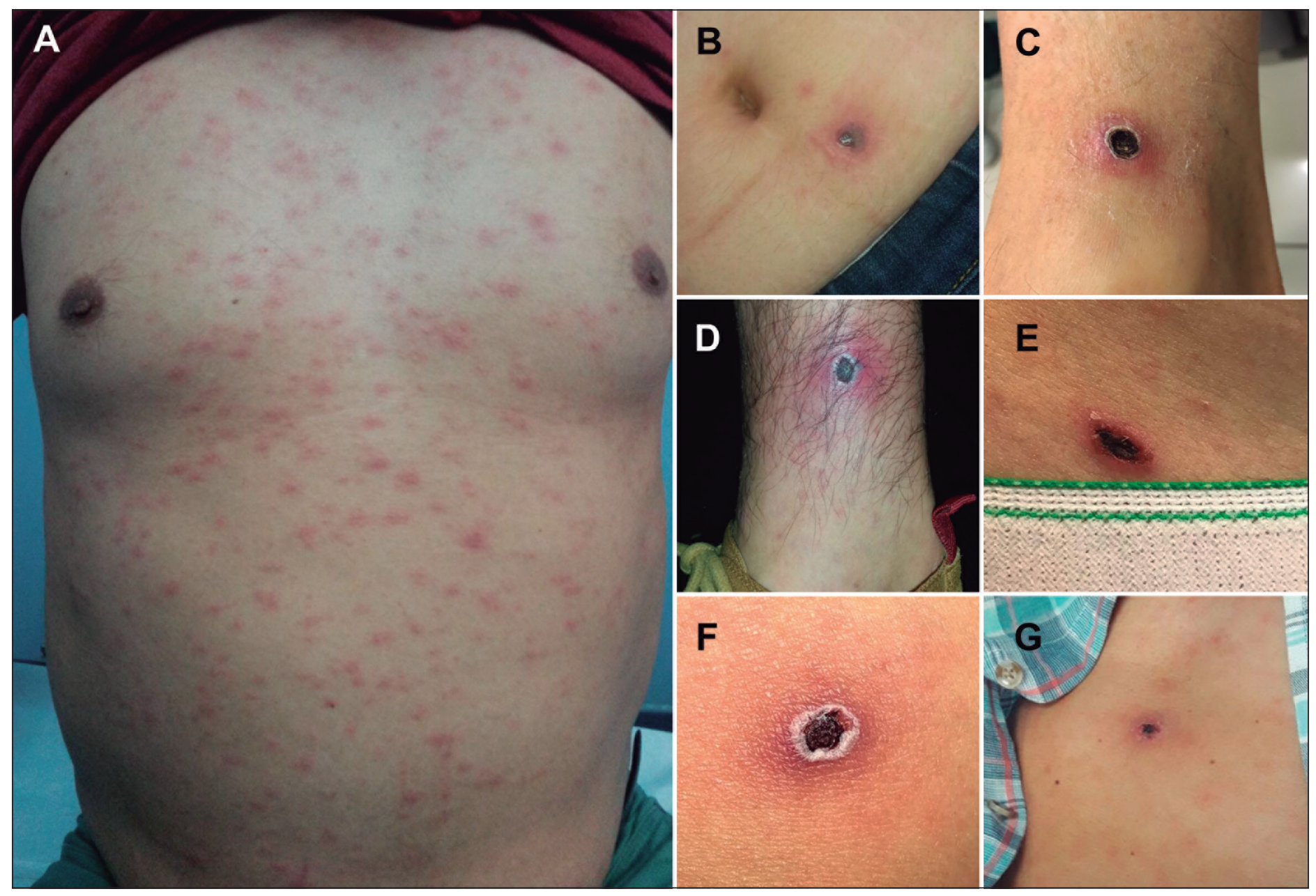

Figura 1. Manifestaciones cutáneas en casos de tifus de los matorrales ocurridos en Chile. A: El típico exantema es maculo-papular grueso, comienza en el tronco y no afecta palmas, plantas ni mucosas. B-G: Las lesiones de inoculación son patognomónicas. Se trata de una escara necrótica profunda, adherente, pequeña (5-10 mm) y poco dolorosa. 
y escarlatina quirúrgica, lo que ha llevado a uso de varios antimicrobianos, anti histamínicos, y retardo en el inicio de la terapia con doxiciclina. Con el uso de este antimicrobiano, todos los pacientes han experimentado una rápida mejoría de la fiebre y de los síntomas constitucionales, persistiendo en algunos de ellos fatiga y cefalea por varios días.

El diagnóstico de esta y otras rickettsiosis es fundamentalmente clínico, y sustenta el inicio de la terapia apropiada y precoz, la que mejora el pronóstico del paciente $^{12}$. Junto a ello, y en especial en zonas donde la epidemiología no está completamente establecida, como es el caso de nuestro país, se recomienda siempre confirmar la sospecha clínica mediante exámenes de laboratorio, que se encuentran disponibles en Chile en el marco de las investigaciones en curso.

Los ensayos diagnósticos incluyen la detección de anticuerpos séricos por distintas técnicas, la detección de material genético por reacción de polimerasa en cadena (RPC) y el cultivo ${ }^{11}$. El comportamiento de las diversas técnicas serológicas varía dependiendo de varios factores, entre ellos la cepa presente en cada localidad ${ }^{18,19}$. El cultivo requiere de instalaciones de bioseguridad III, por lo que la RPC constituye el mejor método de confirmación, siendo el material de la escara la muestra más recomendada, pues además de otorgar la mayor sensibilidad, permanece positiva aún varios días después de iniciado el tratamiento antimicrobiano $^{20,21}$. Otros materiales para el diagnóstico

\begin{tabular}{|l|l|}
\hline Tabla 1. Puntos claves de fiebre de los matorrales sudamericana \\
\hline Antecedentes & $\begin{array}{l}\text { - Actividades recreacionales o laborales en zonas rurales en- } \\
\text { démicas (contacto con vegetación o leña) }\end{array}$ \\
\hline Manifestaciones & $\begin{array}{l}\text { - Triada típica: } \\
\text { clínicas }\end{array}$ \\
& $\begin{array}{l}\text { 2. Enfermedad febril aguda } \\
\text { 3. Exantema maculo-papular: tronco más que extremidades y cara } \\
\text { (no afecta plantas, palmas ni mucosas) } \\
\text { - Frecuente: calofríos, sudoración nocturna, cefalea, mialgias, fatiga } \\
\text { - Menos frecuente: compromiso cualitativo de conciencia, inyección } \\
\text { conjuntival }\end{array}$ \\
- Frecuente: aumento de transaminasas hepáticas y reactantes de fase \\
aguda \\
laboratorio
\end{tabular}

molecular incluyen un hisopado de la base de la escara y el buffy coat (leucocitos separados de sangre con EDTA).

El estudio actualmente en curso por nuestro grupo incluye entre sus objetivos identificar la mejor técnica serológica para nuestro país, implementar el cultivo y optimizar la RPC. Asimismo, se está estudiando el espectro clínico y la extensión geográfica de la infección, la identificación del vector y la secuencia genética de la bacteria identificada en Chile de modo de confirmar si se trata de O. tsutsugamushi o especie(s) relacionada(s). Todos estos aspectos se encuentran en proceso al momento de esta publicación.

Como resultado de los primeros casos, el Ministerio de Salud emitió una alerta epidemiológica en marzo de 2016 para la Región de Los Lagos (Ordinario B51:632), y para todo el país en diciembre de 2016 (Ordinario B51:414022), indicando la necesidad de notificar los casos a la Secretaría Regional Ministerial (SEREMI) de Salud respectiva y el envío de muestras al equipo investigador, puesto que no se encuentran disponibles exámenes diagnósticos como prestación regular en el país. El estudio de laboratorio como parte del proyecto de investigación estará disponible hasta marzo de 2021 (FONDECYT Regular N¹170810).

Hasta la fecha, Chile es el único país de América donde ha sido confirmada esta rickettsiosis ${ }^{23,24}$. Sus hallazgos clínicos son característicos, existe una terapia específica de alta eficacia que no corresponde a los antimicrobianos de uso habitual en cuadros febriles. Los casos no tratados oportunamente pueden desarrollar complicaciones e incluso la muerte. Todo eso enfatiza la importancia que los médicos clínicos que se desempeñan en Chile conozcan esta entidad, puedan sospecharla precozmente e iniciar la terapia adecuada a la brevedad. (Tabla 1). La toma de muestras y su envío al equipo investigador es relevante para comprender mejor la enfermedad, pero no debe retrasar el inicio de la terapia, pues el diagnóstico es eminentemente clínico.

\section{Mensajes clave:}

- El tifus de los matorrales es una nueva enfermedad endémica en Chile que causa infecciones con un potencial fatal.

- Los médicos en Chile deben conocer las manifestaciones típicas de esta infección para plantear el diagnóstico clínico inicial: fiebre, exantema maculo-papular y escara necrótica negra.

- Doxiciclina (100 mg cada 12 h vía oral, por 7 días) es el tratamiento de elección, está disponible en Chile y tiene un efecto muy rápido y eficiente.

- Se recomienda enviar muestras diagnósticas para confirmar la infección y en caso de dudas (diagnósticas o clínicas) contactar a los investigadores. 


\section{Resumen}

El tifus de los matorrales, causado por Orientia tsutsugamushi y transmitido por larvas de ácaros trombicúlideos de roedores silvestres es una rickettsiosis endémica en el Asia Pacífico. Luego del primer caso identificado en Chile en el año 2006, nuestro grupo ha identificado más de 30 casos a partir del año 2015. Los casos se han presentado con un marcado predominio en meses de verano, y su presentación clínica incluye fiebre, exantema y una mancha negra en el sitio de inoculación. Otros hallazgos frecuentes han sido cefalea intensa, sudoración nocturna, aumento de PCR, VHS y transaminasas hepáticas. La gran mayoría de los pacientes se han diagnosticado en el sur de Chile (principalmente la isla de Chiloé), pero recientemente también se han presentado casos en la Región Metropolitana, en personas volviendo de viajes al sur de Chile. Los médicos clínicos deben estar informados de esta enfermedad emergente en el país, de modo de sospecharla e iniciar terapia empírica con doxiciclina. La confirmación de estos casos con los métodos diagnósticos disponibles en Chile contribuirá a una mejor comprensión del rango epidemiológico y la relevancia clínica de esta infección nueva en el país.

\section{Referencias bibliográficas}

1.-- Balcells M E, Rabagliati R, García P, Poggi $\mathrm{H}$, Oddó D, Concha M, et al. Endemic scrub typhus-like illness, Chile. Emerg Infect Dis 2011; 17: 1659-63. http://dx.doi.org/10.3201/ eid1709.100960

2.- Laval E. Epidemia de tifus exantemático en Chile (1932-1939). Rev Chil Infect 2013; 30: 313-316. http://dx.doi.org/10.4067/S071610182013000300007

3.- Watt G, Parola P. Scrub typhus and tropical rickettsioses. Curr Opin Infect Dis 2003; 16: 429-36. DOI: 10.1097/01. qco.0000092814.64370.70

4.- Kelly D J, Fuerst P A, Ching W M, Richards A L. Scrub typhus: the geographic distribution of phenotypic and genotypic variants of Orientia tsutsugamushi. Clin Infect Dis 2009; 48: S20330. doi: $10.1086 / 596576$

5.- Xu G, Walker D H, Jupiter D, Melby P C, Arcari C M. A review of the global epidemiology of scrub typhus. PLoS Negl Trop Dis. 2017; 11: e0006062. doi: 10.1371/journal. pntd.0006062.

6.- World Health Organization Department of Communicable Disease. Surveillance and Response. WHO Recommended Surveillance Standards 1999; 2: 123-4. Available at: http:// www.who.int/csr/en/

7.- Paris D H, Shelite T R, Day N P, Walker D H. Unresolved problems related to scrub typhus: a seriously neglected life-threatening disease. Am J Trop Med Hyg 2013; 89: 301-7. doi:10.4269/ ajtmh.13-0064

8.- Santibáñez P, Palomar A M, Portillo A, Santibáñez S, Oteo J A. The role of chiggers as human pathogens, an overview of tropical diseases. Amidou Samie, Intech Open, Dec 2015. doi: 10.5772/61978. Available from: https://www.intechopen.com/books/ an-overview-of-tropical-diseases/the-role-ofchiggers-as-human-pathogens.

9.- Salje J. Orientia tsutsugamushi: A neglected but fascinating obligate intracellular bacterial pathogen. PLoS Pathog 2017; 13: e1006657. https://doi.org/10.1371/journal. ppat.1006657

10.- Aggarwal S, Sharma A, Sharma V. Eschar: a cutaneous clue to scrub typhus. Braz J Infect Dis 2012; 16: 407-8. http://dx.doi. org/10.1016/j.bjid.2012.06.014

11.- Koh G C K W, Maude R J, Paris D H, Newton P N, Blacksel S D. Review: diagnosis of scrub typhus. Am J Trop Med Hyg 2010; 82: 36-370. doi:10.4269/ ajtmh.2010.09-0233

12.- Wee I, Lo A, Rodrigo C. Drug treatment of scrub typhus: a systematic review and metaanalysis of controlled clinical trials. Trans R Soc Trop Med Hyg. 2017; 111: 336-44. doi: 10.1093/trstmh/trx066.

13.- Kim Y S, Kim D M, Yoon N R, Jang M S, Kim C M. Effects of rifampin and doxycycline treatments in patients with uncomplicated scrub typhus: an open-label, randomized, controlled trial. Clin Inf Dis 2018; ciy130. doi: 10.1093/ cid/ciy 130

14.- Kim D M, Kim S W, Choi S, Yun N R. Clinical and laboratory findings associated with severe scrub typhus. BMC Infectious Diseases 2010; 10: 108. http://www. biomedcentral.com/1471-2334/10/108

15.- Taylor A J, Paris D H, Newton P N. a systematic review of mortality from untreated scrub typhus (Orientia tsutsugamushi). PLoS Negl Trop Dis 2015; 9: e0003971. doi:10.1371/ journal.pntd.0003971

16.- Bonell A, Lubell Y, Newton P N, Crump J A, Paris D H. Estimating the burden of scrub typhus: A systematic review. PLoS Negl Trop Dis 2017; 11: e0005838. doi.org/10.1371/ journal.pntd.0005838

17.- Weitzel T, Dittrich S, López J, Phuklia W, Martinez-Valdebenito C, Velásquez K, et al. Endemic scrub typhus in South America. N Engl J Med 2016; 375: 954-61. doi: 10.1056/ NEJMoa1603657
18.- Blacksell S D, Tanganuchitcharnchai A, Nawtaisong P, Kantipong P, Laongnualpanich A, Day N P J, et al. Diagnostic accuracy of the InBios Scrub Typhus Detect EnzymeLinked Immunoassay for the detection of IgM antibodies in Northern Thailand. Clin Vaccine Immunol 2016; 23: 148-54. doi:10.1128/ CVI.00553-15.

19.- Blacksell S D, Bryant N J, Paris D H, Doust J A, Sakoda Y, Day N P. Scrub typhus serologic testing with the indirect immunofluorescence method as a diagnostic gold standard: a lack of consensus leads to a lot of confusion. Clin Infect Dis 2007; 44: 391-401. https://doi.org/10.1086/510585.

20.- Kim D M, Kim H L, Park C Y, Yang T Y, Lee J H, Yang J T, et al. Clinical usefulness of eschar polymerase chain reaction for the diagnosis of scrub typhus: a prospective study. Clin Infect Dis 2006; 43: 1296-300. https://doi. org/10.1086/508464.

21.- Le Viet N, Laroche M, Thi Pham H L, Viet N L, Mediannikov O, Raoult D, et al. Use of eschar swabbing for the molecular diagnosis and genotyping of Orientia tsutsugamushi causing scrub typhus in Quang Nam province, Vietnam. PLoS Negl Trop Dis 2017; 11: e0005397. doi:10.1371/journal. pntd.0005397.

22.- Available at link: http://epi.minsal.cl/ wp-content/uploads/2018/05/Ordinario_ B51N4140_Dic2016_TodoPa\%C3\%ADs_ Detecci\%C3\%B3n_casos_de_Tifus.pdf

23.- Xu G, Walker D H, Jupiter D, Melby P C, Arcari C M. A review of the global epidemiology of scrub typhus. PLoS Negl Trop Dis. 2017 Nov 3;11(11):e0006062. doi: 10.1371/journal.pntd.0006062. eCollection 2017 Nov.

24.- Bonell A, Lubell Y, Newton P N, Crump J A, Paris D H. Estimating the burden of scrub typhus: A systematic review. PLoS Negl Trop Dis. 2017; 11(9): e0005838. doi: 10.1371/ journal.pntd.0005838. eCollection 2017 Sep. 